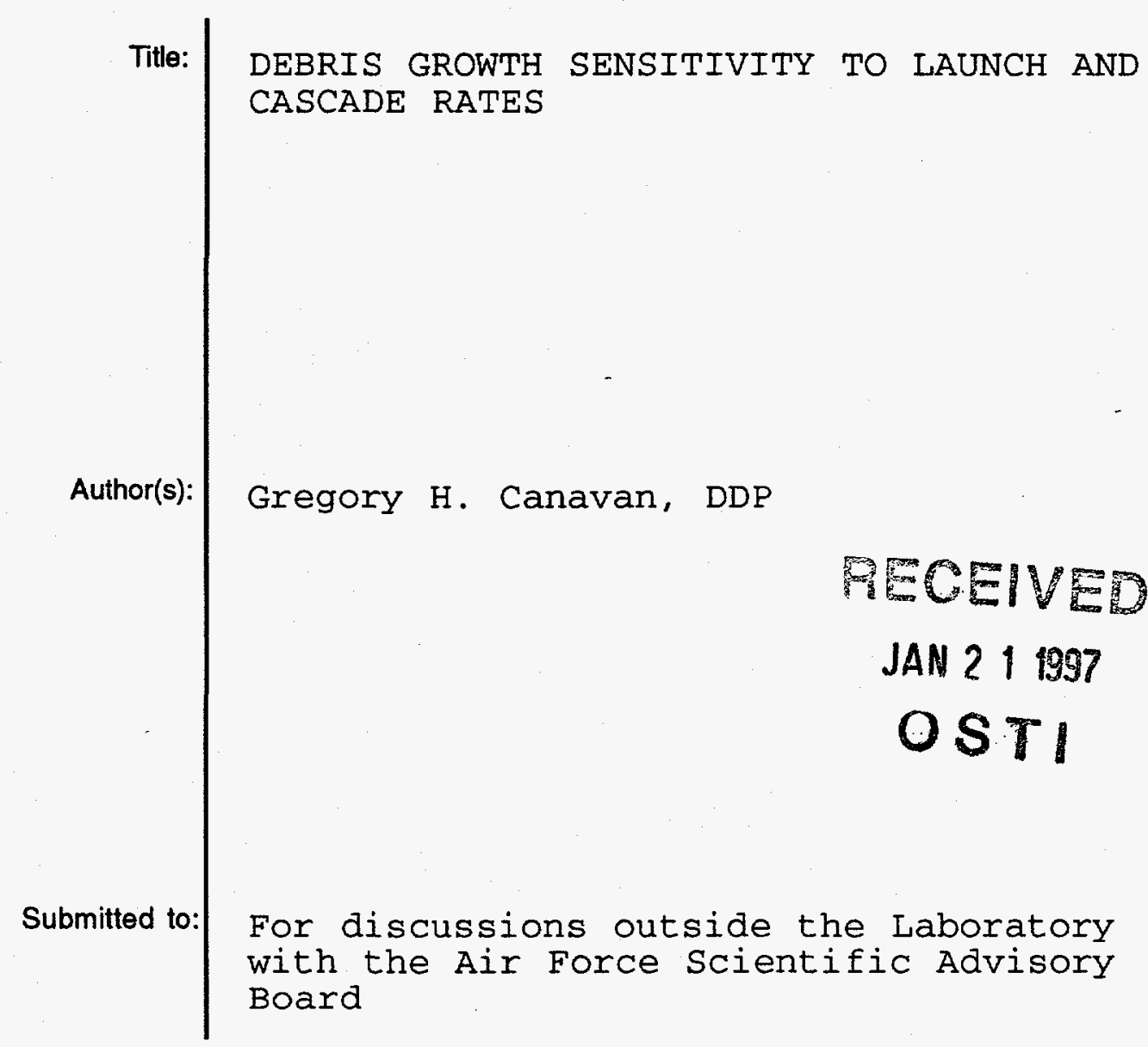

DISCLAIMER

This report was prepared as an account of work sponsored by an agency of the United States Government. Neither the United States Government nor any agency thereof, nor any of their employees, makes any warranty, express or implied, or assumes any legal liability or responsibility for the accuracy, completeness, or usefulness of any information, apparatus, product, or process disclosed, or represents that its use would not infringe privately owned rights. Reference herein to any specific commercial product, process, or service by trade name, trademark, manufacturer, or otherwise does not necessarily constitute or imply its endorsement, recommendation, or favoring by the United States Government or any agency thereof. The views and opinions of authors expressed herein do not necessarily state or reflect those of the United States Government or any agency thereof.

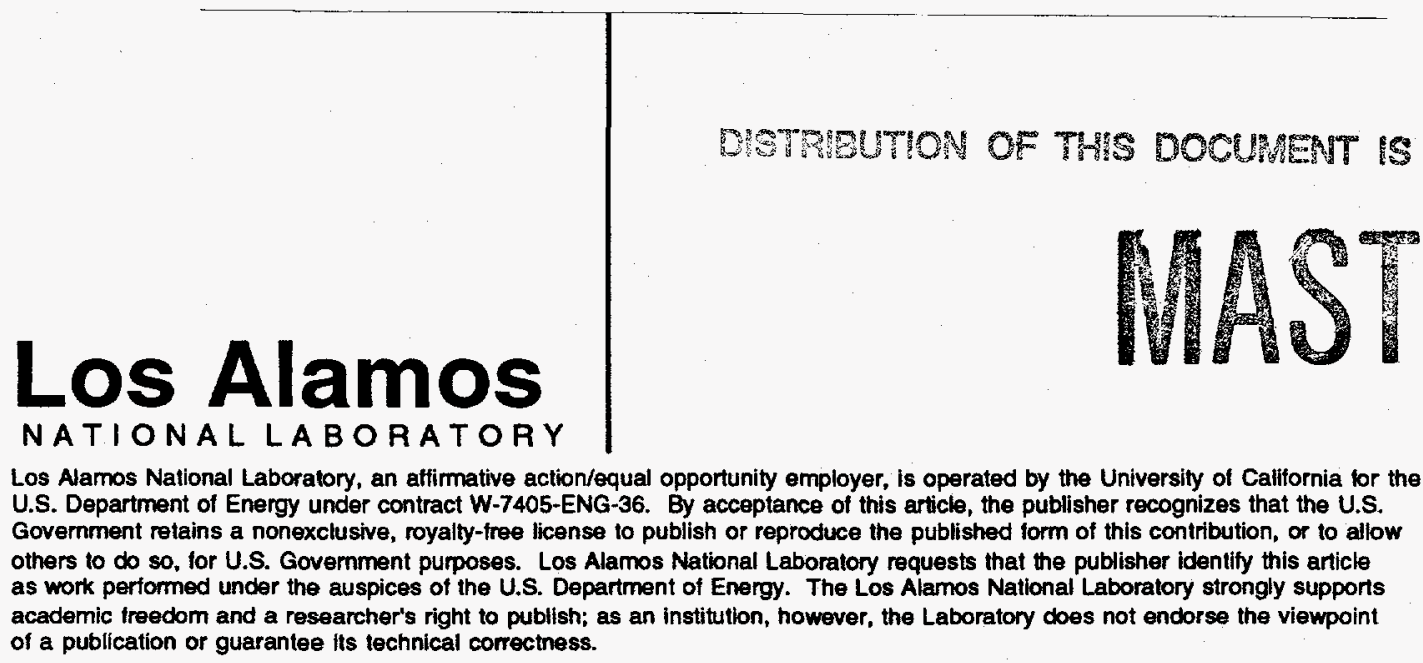




\title{
DEBRIS GROWTH SENSITIVITY TO LAUNCH AND CASCADE RATES
}

\author{
Gregory H. Canavan
}

Two-component models provide a good description of debris growth from the outset of launch to the present, predictions of future trends, and assessments of their sensitivity. Launch rate reductions produce less than proportional reductions in debris, for reasons that are discussed. The shift of debris to higher altitudes is assessed quantitatively, although the details of the growth are discussed elsewhere.

Launch rate projections from an earlier paper are used in a two-component model to provide a good description of debris growth from the initiation of high launch rates to the present. They can also be used to discuss the sensitivity of those projections to the sources of fragments and intact objects that produce the current catalog distribution and to changes in launch rate. Debris growth is sensitive to launch rate, because the number of objects and fragmentations per launch has historically been fairly stable, so launch rate also determines the rate of production of debris on orbit. The model predicts current conditions quite accurately and a relatively unsurprising extrapolation of those results for historical launch rates and altitude profiles. Projected two to four-fold reduction in launch rates would produce roughly proportional reductions in debris, although the time scales for decay of objects from the upper peak make that simple equilibrium estimate optimistic by about a factor of two.

For reduced launch rates, historical sources and orbital decay both tend to shift the preponderance of debris to higher altitudes. For a four-fold reduction, the increase is about a factor of two. For zero sources, the shift is about a factor of four, which is accomplished primarily by the elimination of fragments at low altitudes. The persistence of the debris peak at $1,450 \mathrm{~km}$ primarily reflects the long time scales for decay of either intact objects or fragments there. Two component models appear adequate for the evaluation of sensitivity to launch rate and debris spectrum at current or reduced launch rates. They are shown elsewhere to be adequate for the discussion of high launch and cascade rates and strong sources at high altitudes.

A companion note ${ }^{1}$ shows that a two-component model provides a reasonable description of the growth of debris from the initiation of high launch rates $\approx 35$ years ago to the present. It also discusses the sources of fragments and intact objects that produce the current catalog distribution. Historical launch and fragmentation rates provide an estimate of the altitude distribution of debris sources. In the model, fragments and intact objects are each described by a set of time dependent equations for the evolution of the densities in $100 \mathrm{~km}$ altitude bins, using appropriate sources ${ }^{2}$ and average decay times for each. ${ }^{3}$ As launch rate variations of interest 
resemble the overall variations experienced in the past, it would appear that a flux model should be adequate for a preliminary survey of sensitivity of debris growth to launch and cascade rates.

Current conditions. The previous note used predicted fragment spectra and the current observed distribution of spacecraft, respectively, as surrogates for the future sources of fragments and intact objects. While that approached proved adequate, this note uses the more fundamental approach of sorting the debris launch catalog. 4 Figure 1 compares the fragment and large object densities predicted by integrating the model equations forward from zero objects 35 years ago to the fragment and large object densities measured in the current catalog. The predicted values match the debris catalog is within about 5 to $10 \%$ at and between the two peaks at 950 and 1,450 $\mathrm{km}$, which is the region that contains most of the debris density and which is the greatest concern for cascading. The flux model calculations match the detailed spectrum from the peak at $950 \mathrm{~km}$ through the minimum at $1,250 \mathrm{~km}$ to the second peak at $1,450 \mathrm{~km}$ and at all higher altitudes.

At altitudes below $800 \mathrm{~km}$ the model densities fall more rapidly than the catalog.

Presumably, this is due to the simplified treatment of eccentricity used, which underestimates the lifetimes of both fragments and intact objects at low altitudes. However, the discrepancy is less than a factor of two for all altitudes at which debris growth is a serious concern. The greatest fractional discrepancy is about $40 \%$ at $750 \mathrm{~km}$. That altitude is somewhat low for significant cascading. Below $700 \mathrm{~km}$ most of the surviving objects are large intact objects which decay slowly. Fragments, which are assumed to have an average area $<0.3 \mathrm{~m}^{2}$ and an areal density of $\geq 3 \mathrm{~kg} / \mathrm{m}^{2}$ are rapidly removed there, while the large objects, which have an average area of $\approx 10$ $\mathrm{m}^{2}$ and an areal density of $150 \mathrm{~kg} / \mathrm{m}^{2}$, typically have lifetimes of years to decades.

Continuation of current rates. Figure 2 shows the large object, fragment, and total densities that result from projecting these historical growth rates 200 years from 1960 , which is taken to be the start of high launch rates. The distribution at 35 years-i.e., today-is that shown in Fig. 1. The bottom line is for large, intact objects; the next is for fragments; the top line is their sum. The model correctly predicts that the total number of debris objects would grow to about 5,270 today, and that it would grow from there to about 20,000 in 2160 . Fragments would grow to 14,860 ; large objects to 5,600. The growth rate is highest in the first few decades. The total reaches about 10,000 by the end of the first century, and doubles in the second century, which corresponds to $\approx 1 \% / \mathrm{yr}$ growth in the first century and $0.7 \% / \mathrm{yr}$ in both.

Growth is essentially due to launch and explosion, as offset by orbital decay. These sources and sinks come into a rough balance by the end of the first century. Launch and explosion produce a total of about 88,000 objects over the 200 years, of which about 68,000 decay to the ground and 20,000 remain on orbit. Collisional cascading contributes $\approx 900$ objects, or $1 \%$ of the 88,000 objects launched into or produced in orbit. 
Figure 3 shows the distribution of objects over altitude at year 200 , or 2165 . The distribution still has the two peaks of Fig. 1. Each has grown by about a factor of three, and the minimum between them has partially filled in. The large objects of year 200 alone could fill the catalog of today. Because of their longer lifetime, the large objects constitute most of the low altitude part of the lower peak. Because of their higher production rate, the fragments constitute most of its upper part. Thus, fragments make a smaller relative contribution to the $950 \mathrm{~km}$ peak, although the relative contributions from fragments and large objects are about the same at year 200 as at present.

Each of these trends is expected. The increase of the peaks is essentially linear due to the use of historic launch and fragmentation rates. That the peak at $1,450 \mathrm{~km}$ grows more than the one at $950 \mathrm{~km}$ is due to a combination of the large sources and low drag there. While its advantage in drag is fundamental, that due to sources is not. They reflect the large historical Soviet launch rates to that altitude for missions that are no longer necessary or supported. If those launch rates were reduced, the growth of the $1,450 \mathrm{~km}$ peak would be reduced proportionally. Fragments make a smaller relative contribution to the peak at $950 \mathrm{~km}$ in year 200 than now because the large objects have comparable source rates and lower drag there. Fragments make a comparable contribution to intact objects in the peak at 1,450 because neither they nor the large objects experience much drag there. As to the minimum between the two peaks increasing, at $1,250 \mathrm{~km}$ the source is about $0.6+5.1=5.7$ fragments per year, and decay losses are small, so the difference of 5 fragments/year over 200 years accumulates to the $\approx 1,000$ fragments shown.

Projected rates. The launch rates used in Figs. 1 to 3 are high relative to projections for the next century for two reasons. The first is the factor-of-two decrease in the CIS launch rate due to the discontinuation of launches no longer needed after the cold war. The second is the progressive shift of U.S. and CIS payloads from LEO to GEO, which is now about $50 \%$ complete, and continues about linearly in time. Planned communication constellations of 100 to 1000 satellites could maintain launch rates at about $1 / 2$ to $1 / 4$ of the above rates. Were it not for them, launch rates to LEO could drop to $10 \%$ of previous decade's.

Figure 4 shows the growth of debris from the present for $1 / 2$ the peak launch, fragment, and large object production rates. The total number increases from 5,270 to about 13,000 , about $7,730 / 165$ years $\approx 47$ objects per year, which is about $\approx 0.9 \% / y r$, or about $1 / 3$ the current rate. Large objects increase from 1,240 to 3,000. Fragments increase from 4,030 to 8,000. That is a net increase of $\approx 4,016$ fragments, but that is much smaller than the 12,000 from the projection of historic rates in Fig. 2. At 1/2 the historic rate, a larger fraction of the fragment source is diverted into the decay flux to the ground.

Figure 5 shows the growth of debris from the present for $1 / 4$ the peak launch, fragment, and large object production rates. It resembles Fig. 4, except that the initial decreases in the 


\section{DISCLAIMER}

Portions of this document may be illegible in electronic image products. Images are produced from the best available original document. 
fragment and total density last several decades and their recovery and subsequent growth are slower. The fragment density initially drops $20 \%$; it does not regain its initial value until near the end of the century. The slight growth in the total-about 7,500-5,000 $\approx 2,500$ objects, or 12.5 objects per year-is due primarily to large objects.

Equilibrium response. Since fragments are apparently in equilibrium, i.e., are removed by decay as rapidly as they are produced by explosions, it might be argued that decreasing the launch and fragment production rates by a factor of 4 should decrease the fragment density by a like amount. However, only a fraction of the fragments can be removed on relevant time scales. Figure 6 shows the vertical flux of intact particles, F, its divergence, divF, and the decay time, $\mathrm{N} /$ divF, as functions of altitude at year 200 . The vertical flux is small at high altitudes due to the low drag and sources there. It reaches unity at about $1,550 \mathrm{~km}$. It increases exponentially at lower altitudes, reaching about 100 fragments per year at $250 \mathrm{~km} .5$

The object density at any altitude is reduced by the divergence of the flux, which is the difference between the rates of outflow and inflow of decaying particles at that altitude. At high altitudes, the divergence is about a factor of 3 less than the flux, as there are few sources of particles, and the divergence is roughly equal to the outflow. At and below the peak at $1,450 \mathrm{~km}$, the sources are strong; indeed, at $1,450 \mathrm{~km}$, the source is larger than the outflux, which forces the divergence negative. At lower altitudes, divF increases, but not as rapidly as the flux, which indicates that there is little net loss or gain due to vertical transport. Below $400 \mathrm{~km}$ the divergence increases again due to the large sources there.

The resulting clearing time $\mathrm{N} / \mathrm{divF}$ is small at low altitudes, but increases exponentially. It reaches a decade at about $600 \mathrm{~km}$, a century at about $800 \mathrm{~km}$, and a millennia at about 1,400 $\mathrm{km}$. Because of this rapid increase in clearing time with altitude, whether or not particles can be considered to be in equilibrium depends on their altitude. Particles at $600 \mathrm{~km}$ would respond to reductions in the launch rate in a few decades. Particles at $800 \mathrm{~km}$ would respond in a few centuries. But particles at the upper peak at $1,450 \mathrm{~km}$ would only respond on time scales of thousands of years. That means the particles in the lower peak can be affected by reduced rates, but those in the upper peak cannot.

The current distribution of launches effectively breaks particles into two components. Those at the $950 \mathrm{~km}$ peak and lower can respond to changes in launch rates on time scales of centuries, which is relevant. Those at the $1,450 \mathrm{~km}$ peak cannot. At present, the sources at the two peaks are roughly equal. Thus, reducing the launch rate to $1 / 4$ of its current value would remove the objects below $800 \mathrm{~km}$ in about a century, but it would take much longer to remove those above $1,200 \mathrm{~km}$, so the response of the whole distribution would only be about half that predicted by simple arguments, as seen in Figs. 4 and 5. 
The arguments above are cast in terms of intact fragments, but they apply, with slight modifications, for fragments as well. Orbital decay scales on the product of object areal density and air density. Fragments have areal densities about 30 -fold smaller than intact objects, so the same phenomena apply to fragments at altitudes about 3 scale heights higher than those shown on Fig. 6. At the $950 \mathrm{~km}$ peak and below, the scale height is about $60 \mathrm{~km}$, so that means about $180 \mathrm{~km}$, which does not strongly affect any of the previous arguments. Above $1,000 \mathrm{~km}$ the scale height is about $250 \mathrm{~km}$, so the shift is about $750 \mathrm{~km}$. That means fragments in the upper peak experience drag comparable to intact objects in the lower peak, which is the reason for their greater responsiveness in Figs. 4 and 5.

Altitude distribution. Figure 5 shows the time dependence of the fragments, intact, and all objects for a launch rate $1 / 4$ of current. The large objects grow steadily, the fragments drop for a few decades and then begin to grow faster; and the total increases about $50 \%$ over 200 years. The reasons for those trends can be seen in the altitude distributions of Figs. $7 \mathrm{a}$ to $7 \mathrm{c}$. Figure $7 \mathrm{a}$ shows the altitude distribution of the large objects now and at year 200. Little of the growth is due to cascading; it is mostly due to launch and explosion sources. The peak at $950 \mathrm{~km}$ grows more than that at $1,450 \mathrm{~km}$ because of the larger sources there, as discussed above.

Figure $7 \mathrm{~b}$ shows the growth of the fragments, which is more complicated. The equilibrium fragments on the lower side of the $950 \mathrm{~km}$ peak are strongly eroded at this lower rate, so their peak contribution remains roughly constant, but their integrated contribution falls. By contrast, at $1,450 \mathrm{~km}$, the fragments grows rapidly because of their large source and low drag. However, the sensitivity to the historical rate used is discussed above, and a companion paper discusses the model sensitivity to the fraction of fragments in the upper peak. 6

Figure $7 \mathrm{c}$ shows the total objects. The lower peak is eroded at lower altitudes but otherwise remains about the same size and shape, which conceals the large shift from fragments to large objects at that altitude. The peak at $1,450 \mathrm{~km}$ grows disproportionately, achieving a ratio of about 2 to 1 over the peak at $950 \mathrm{~km}$ by year 200, with comparable contributions from both large objects and fragments, which grow more rapidly for historical launch rates.

Zero sources. Figures $8 \mathrm{a}$ to $8 \mathrm{c}$ show the altitude distribution of intact, fragments, and total debris objects for zero debris sources. Intuitively, one would expect only decay at all altitudes for these conditions. That is not the case here, although some of the results are model dependent. Figure 8 a shows the large objects at present and year 200 . Their distribution appears to be eroded at low altitudes. In fact, the depletion of the distribution is working its way to progressively higher altitudes as time passes. By year 200 , significant depletion is present up to about $800 \mathrm{~km}$, which is in accord with the time scales derived in Fig. 6 . The number of intact objects increases slightly at $1,450 \mathrm{~km}$ in these calculations. That is an artifact of the assumption 
of completely local deposition assumed in these calculations, which is explored in more detail elsewhere and found to be extraneous. 7

Figure $8 \mathrm{~b}$ shows the fragments, which are largely eliminated by orbital decay below $1,000 \mathrm{~km}$, as expected from the scaling arguments above. The erosion appears to extend down to about $1,300 \mathrm{~km}$, although some of the decay from there is offset by the obvious decay from above. The peak at 1,450 again increases, due to model dependent effects that are discussed elsewhere. Figure $8 \mathrm{c}$ shows the total debris objects. The large decrease at low altitudes primarily reflects the near-total elimination of fragments by orbital decay and the gradual elimination of intact objects below about $800 \mathrm{~km}$. The persistence of the peak at $1,450 \mathrm{~km}$ primarily reflects the long time scales for changes by decay there for either intact objects or fragments.

Summary and conclusions. Debris growth is primarily sensitive to launch rate, because for the historically stable number of objects and fragmentations per launch, the launch rate determines the rate of launch of debris objects into orbit. The launch rate projections from an earlier paper can be used in a two-component model to provide a good description of debris growth from the initiation of high launch rates to the present. It can also be used to discuss the sensitivity of those projections to the sources of fragments and intact objects that produce the current catalog distribution and to changes in the launch rate. The model predicts current conditions quite accurately. It also predicts a relatively unsurprising extrapolation of those results for the continuation of historical launch rates and altitudes. Projected two to four-fold reduction in launch rates would produce roughly proportional reductions in debris, although the time scales for decay of objects from the upper peak make that simple equilibrium estimate optimistic by about a factor of two.

For any reduced launch rate, the combination of historical sources and decay tend to shift the preponderance of debris to the upper peak. For a four-fold reduction in launch rate, the increase in 200 years is from rough parity to a two-fold number in the upper relative to the lower. For zero sources, the shift is about a factor of four. It is accomplished by a large decrease at low altitudes, which primarily reflects the near-total elimination of fragments by orbital decay and the gradual elimination of intact objects below about $800 \mathrm{~km}$. The persistence of the peak at 1,450 $\mathrm{km}$ in all cases primarily reflects the long time scales for changes by decay there for either intact objects or fragments. Proper treatment of the deposition of fragments there is essential for quantitative estimates of growth or decay. Overall, two component models of debris decay appear to be adequate for the evaluation of sensitivity to launch rate and debris spectrum at current or reduced launch rates. They are shown elsewhere to be adequate for the discussion of high launch and cascade rates 8 as well as strong sources at high altitudes. 9 
October 24, 1996 6:48 PM

\section{References}

1. G. Canavan, "Estimates of Current Debris from Flux Models," Los Alamos report.

2. G. Canavan, "Debris Masses and Areas Inferred from the Launch Catalog," Los Alamos report, October 1996.

3. G. Canavan, "Averaging Decay Time and Rate Over Fragment Parameters," Los Alamos report, LA-UR-96-2477, Júly 1996.

4. G. Canavan, "Rates Inferred from the Space Debris Catalog," Los Alamos report LA-UR-962803, August 1996.

5. G. Canavan, "Vertical Transport and Sources in Flux Models," Los Alamos report, LA-UR96-2477, July 1996.

6. G. Canavan, Cascade Stability of the Debris Catalog Under a Two-Component Flux Model," Los Alamos report, August 1996.

7. G. Canavan, Cascade Stability of the Debris Catalog Under a Two-Component Flux Model," op cit.

8. G. Canavan, O. Judd, and F. Naka, "Comparison of Space Debris Estimates," Los Alamos report, October 1996.

9. G. Canavan, Cascade Stability of the Debris Catalog Under a Two-Component Flux Model," op cit. 


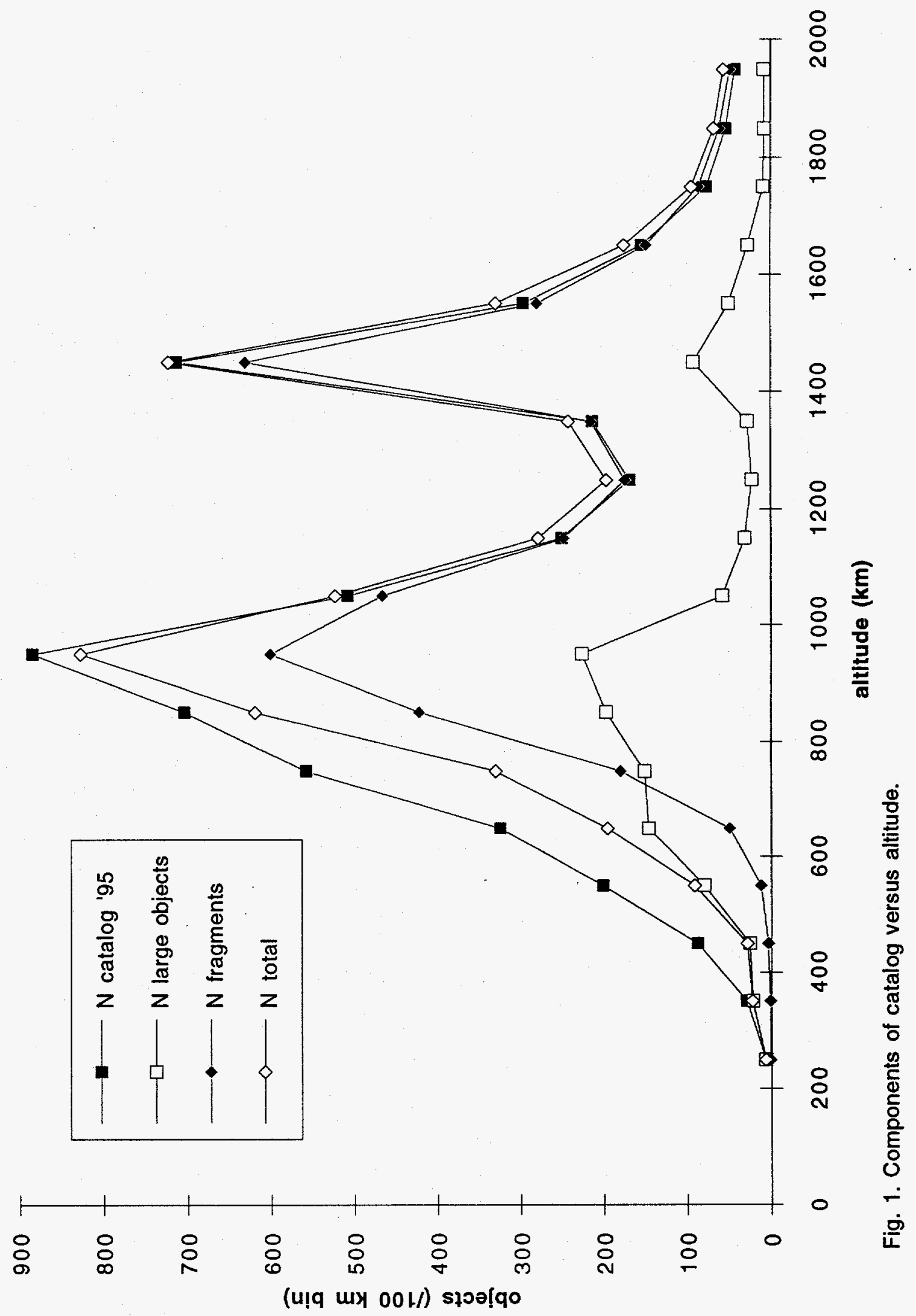




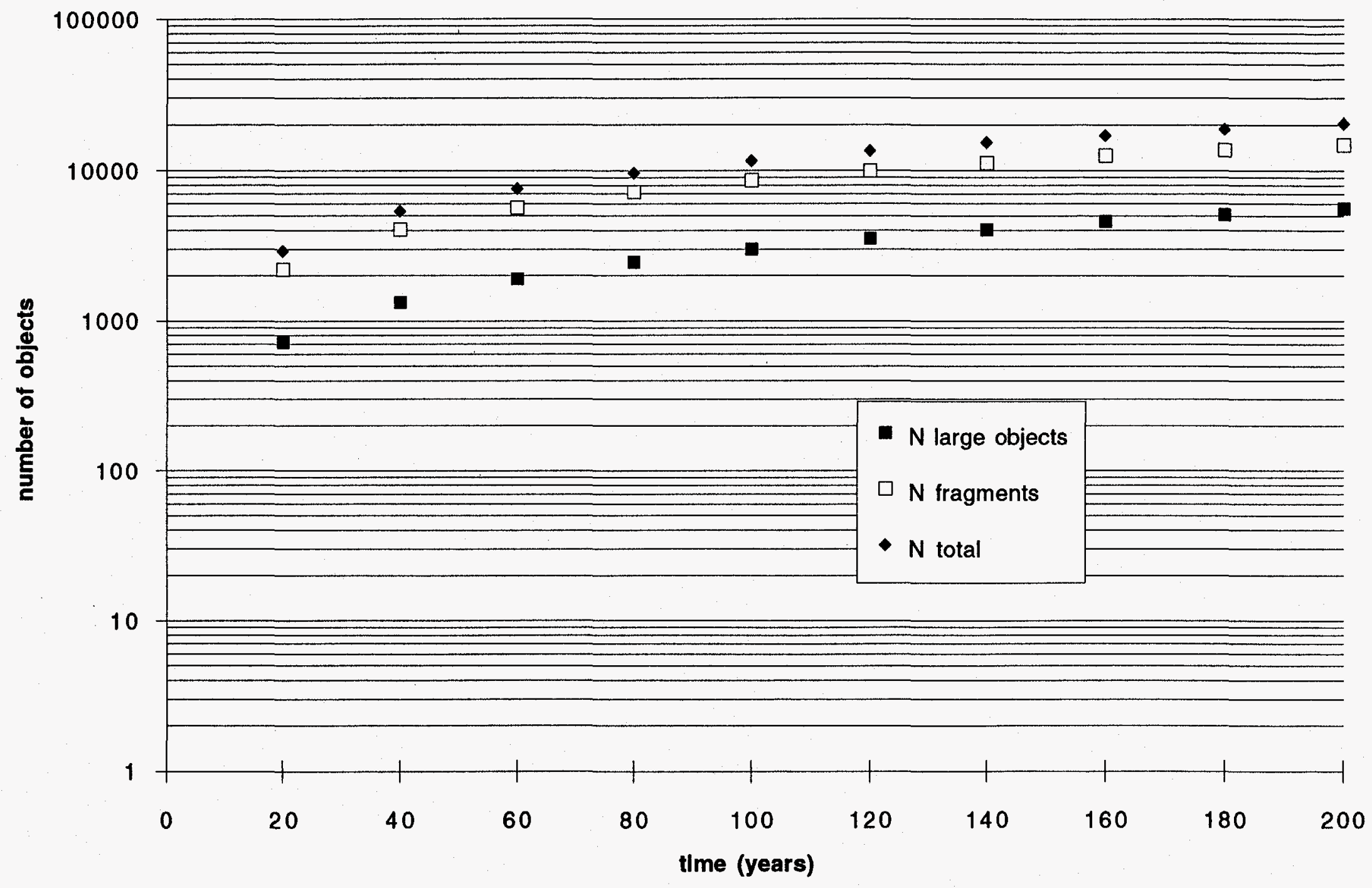

Fig. 2. Fragment, large, and total number versus time. 


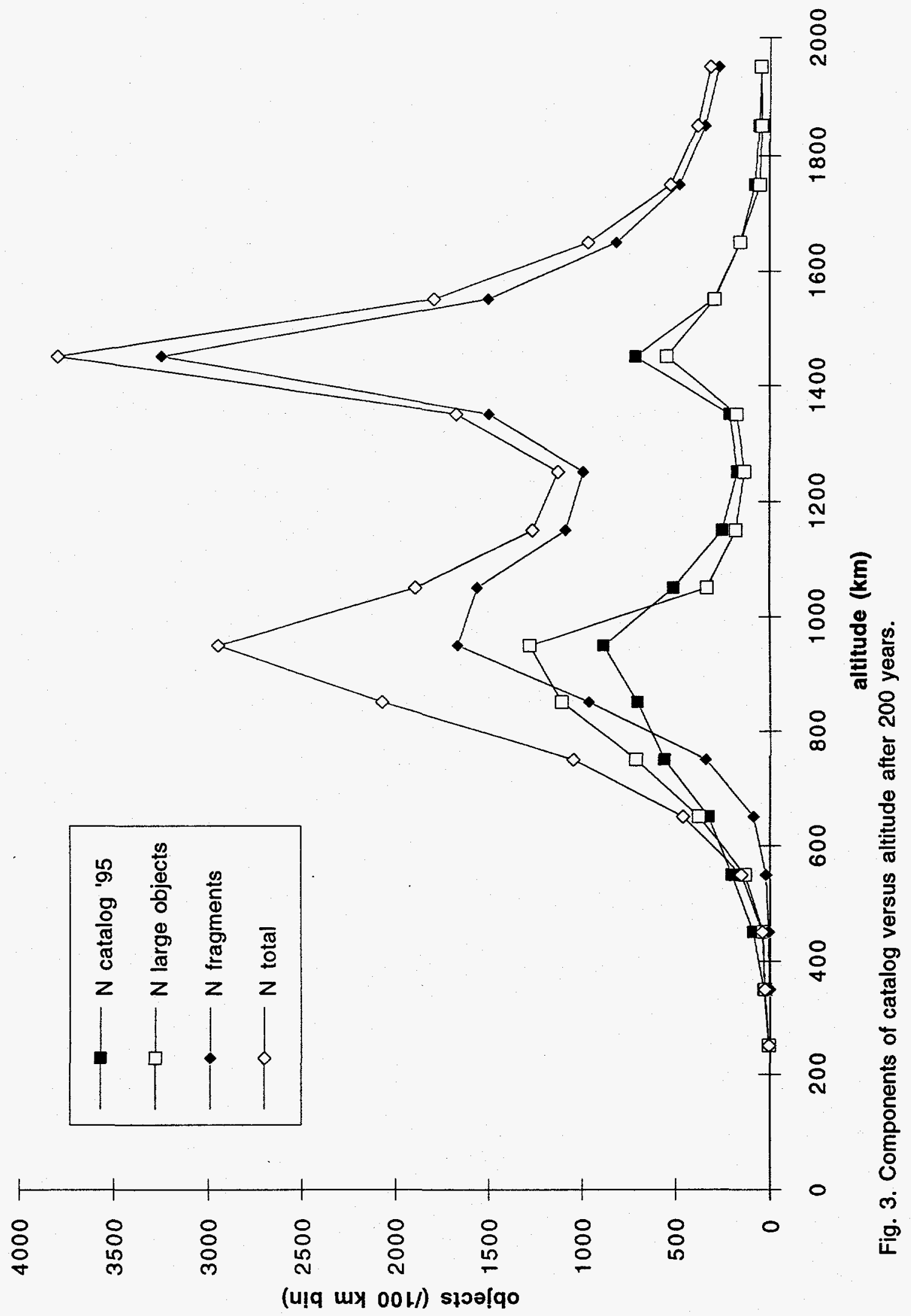




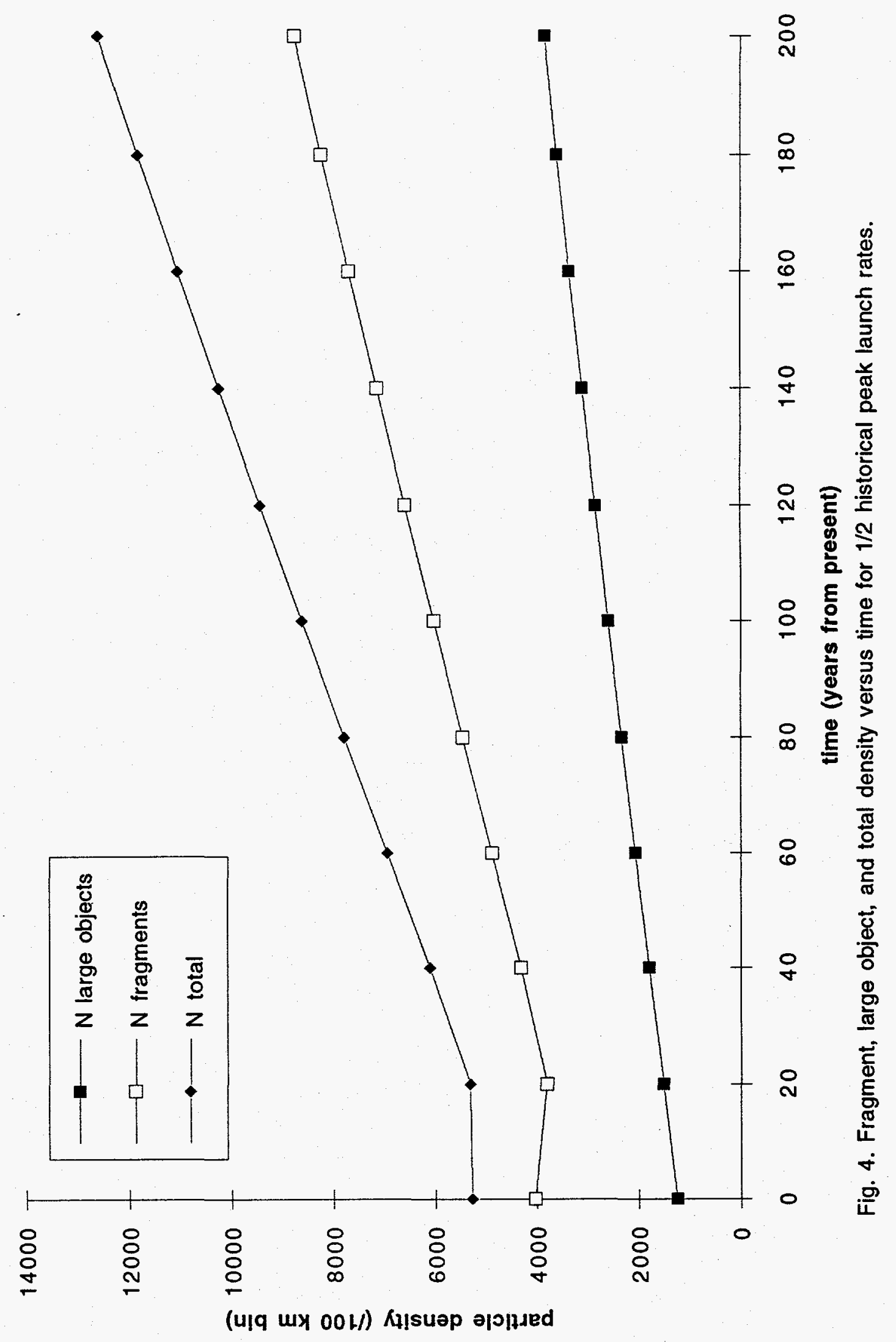




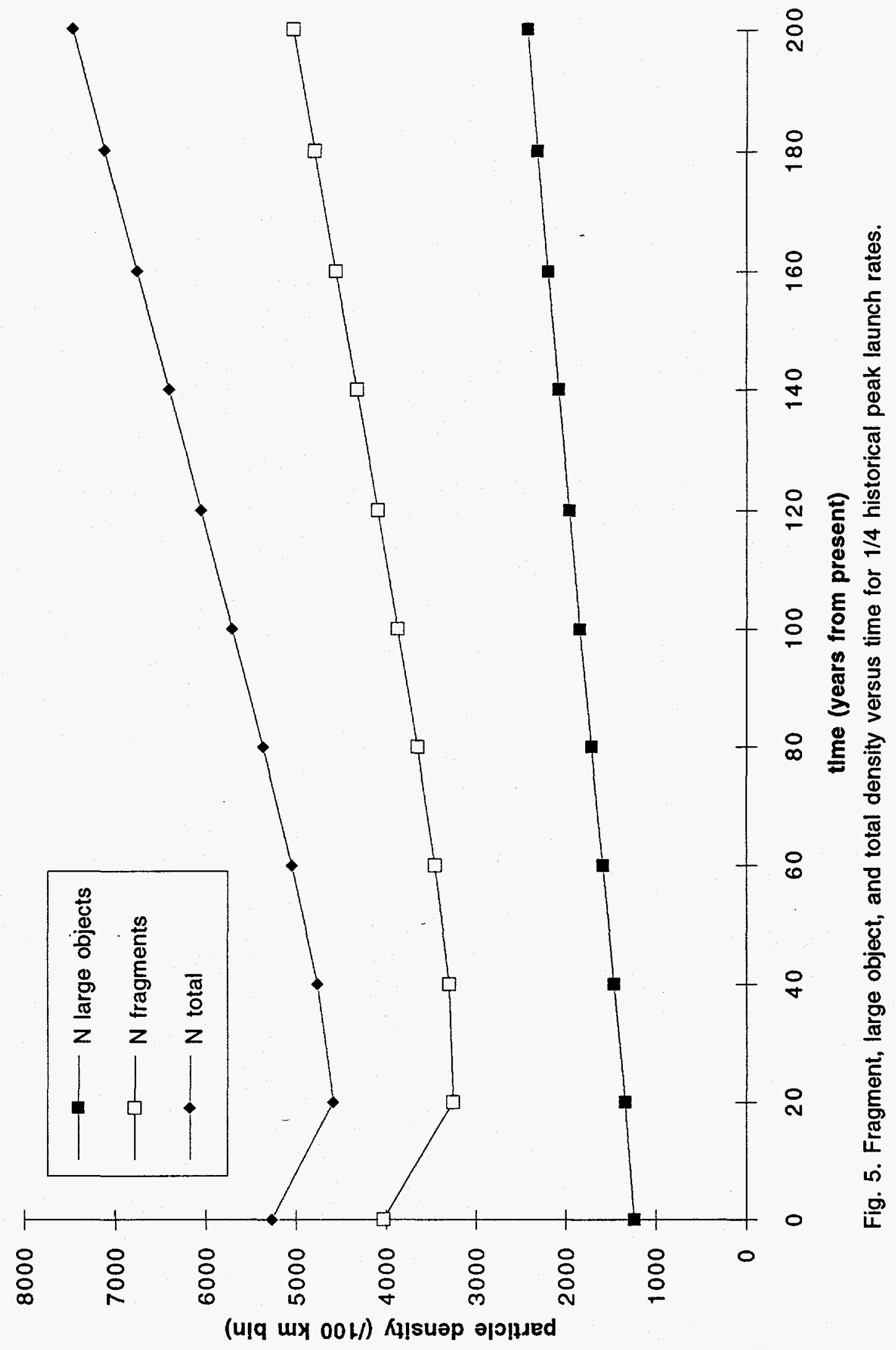




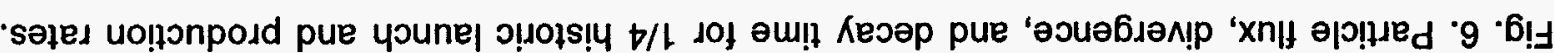

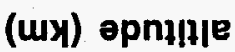

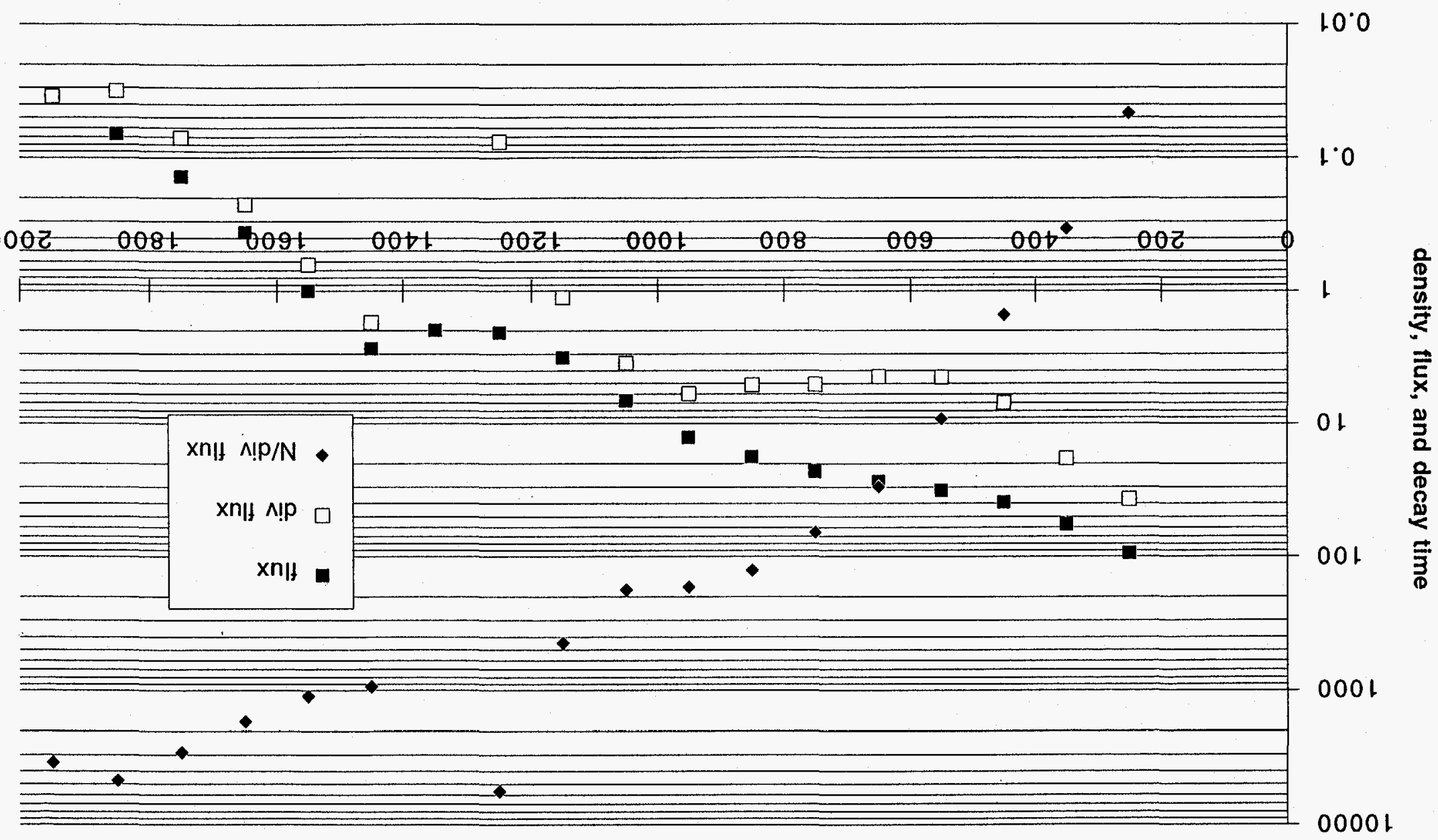




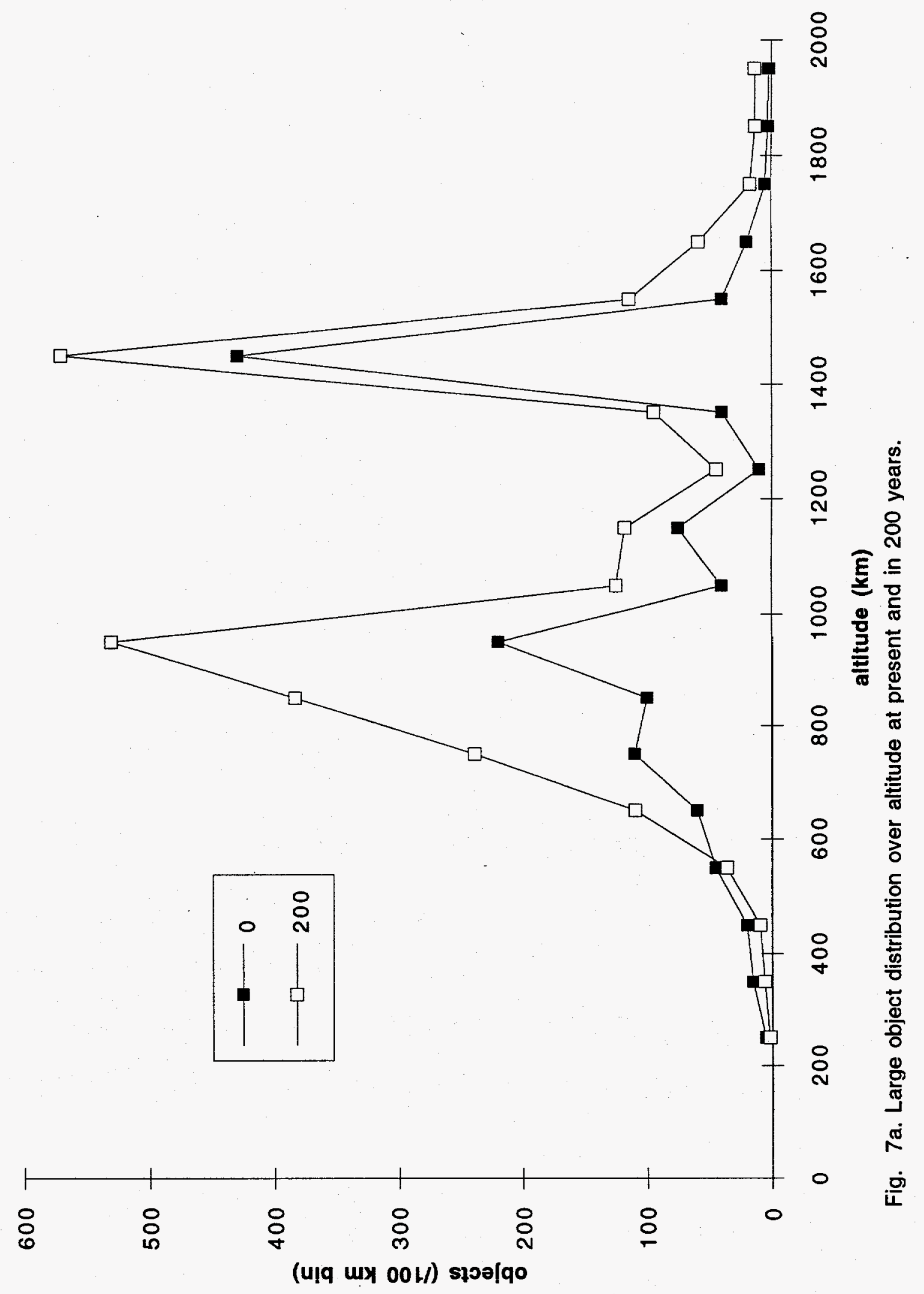




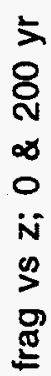

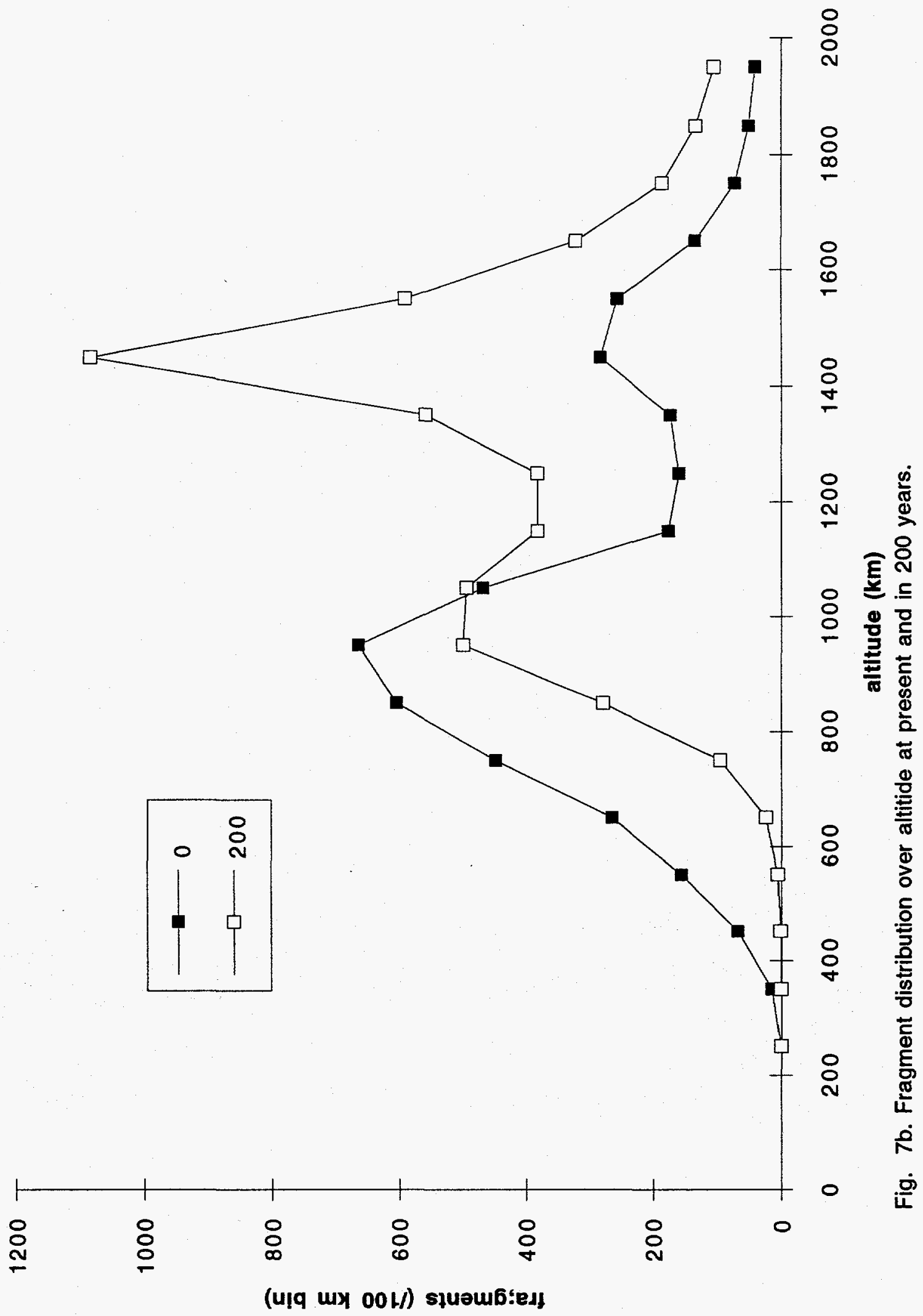




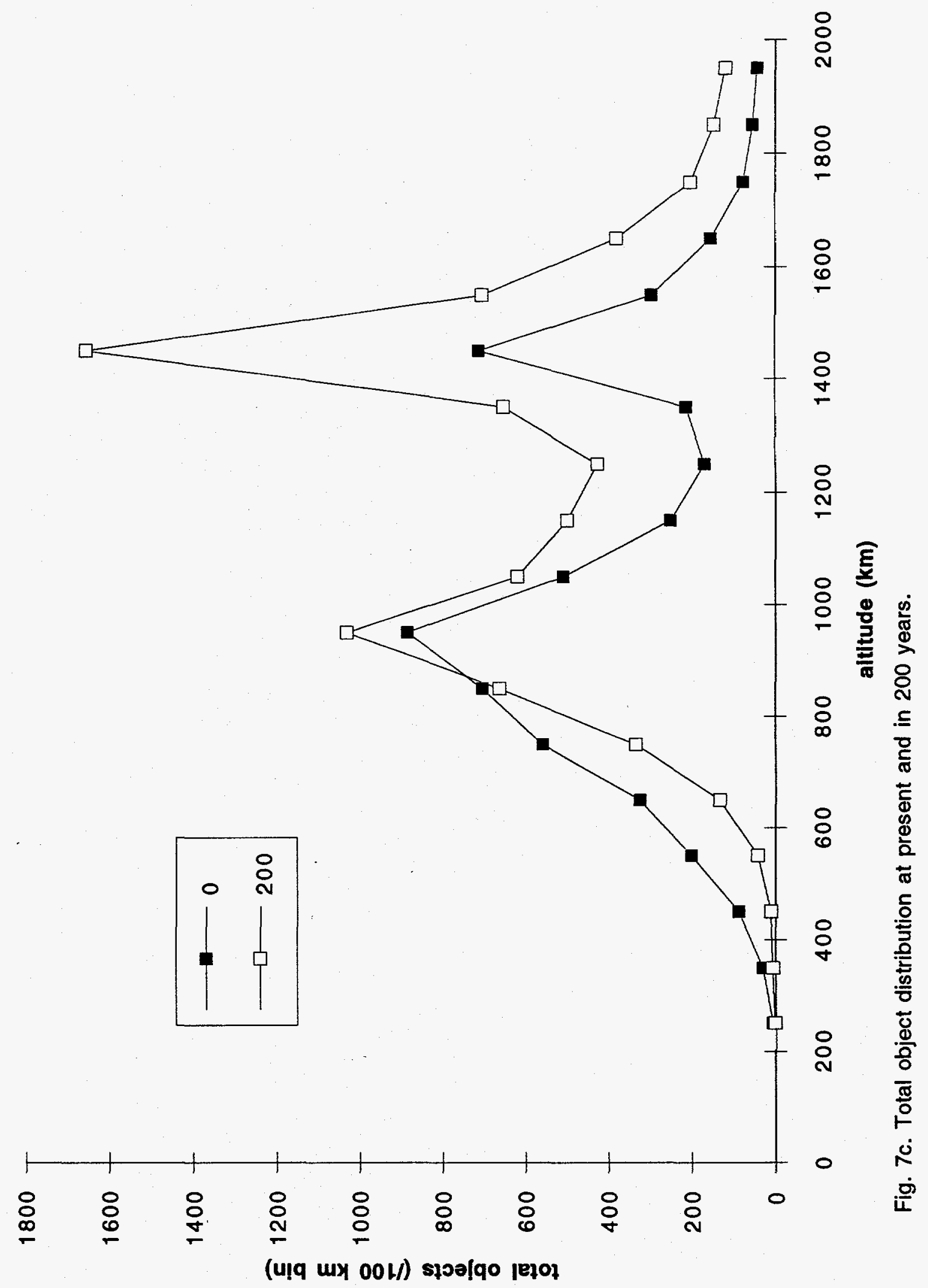




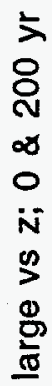

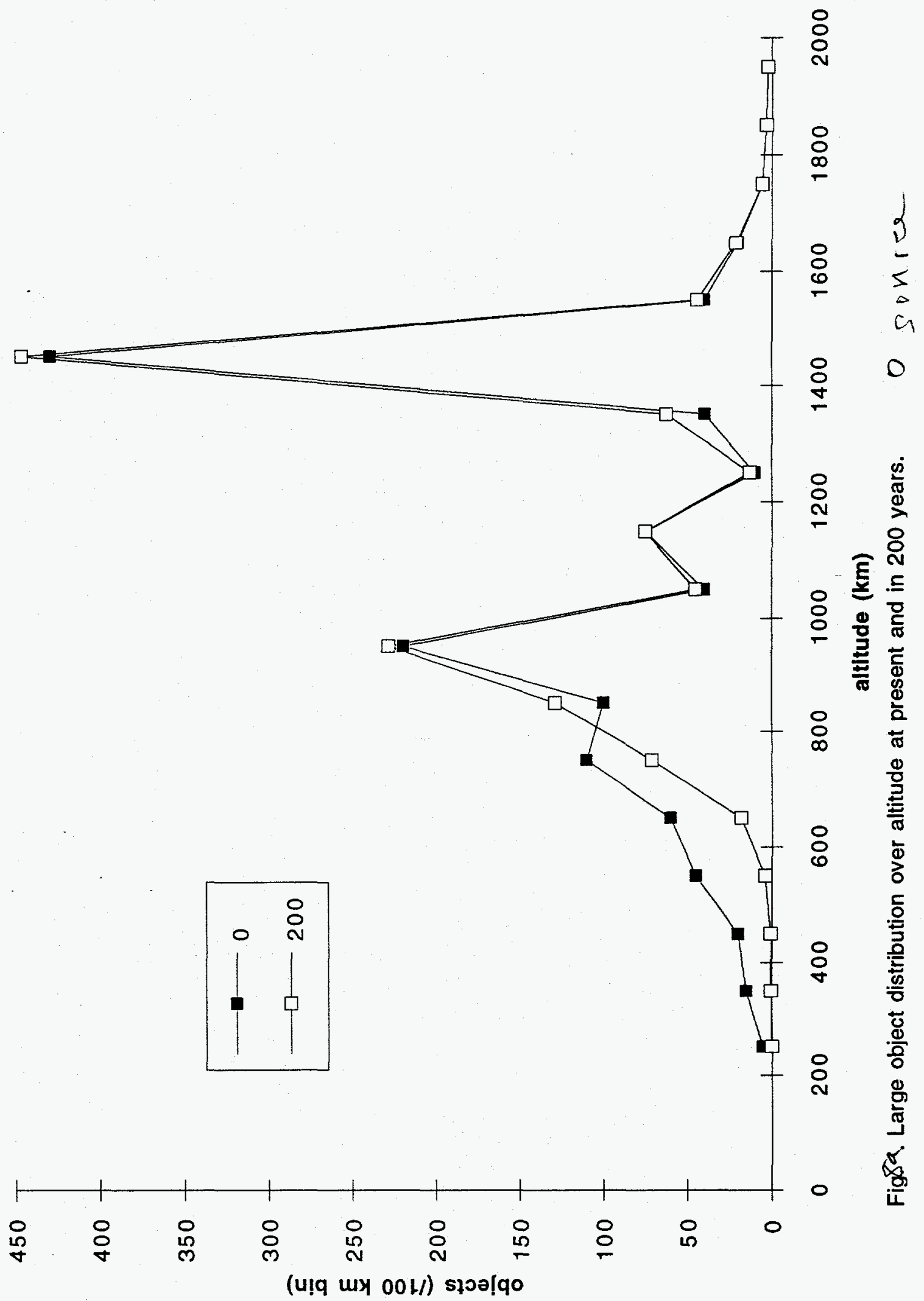




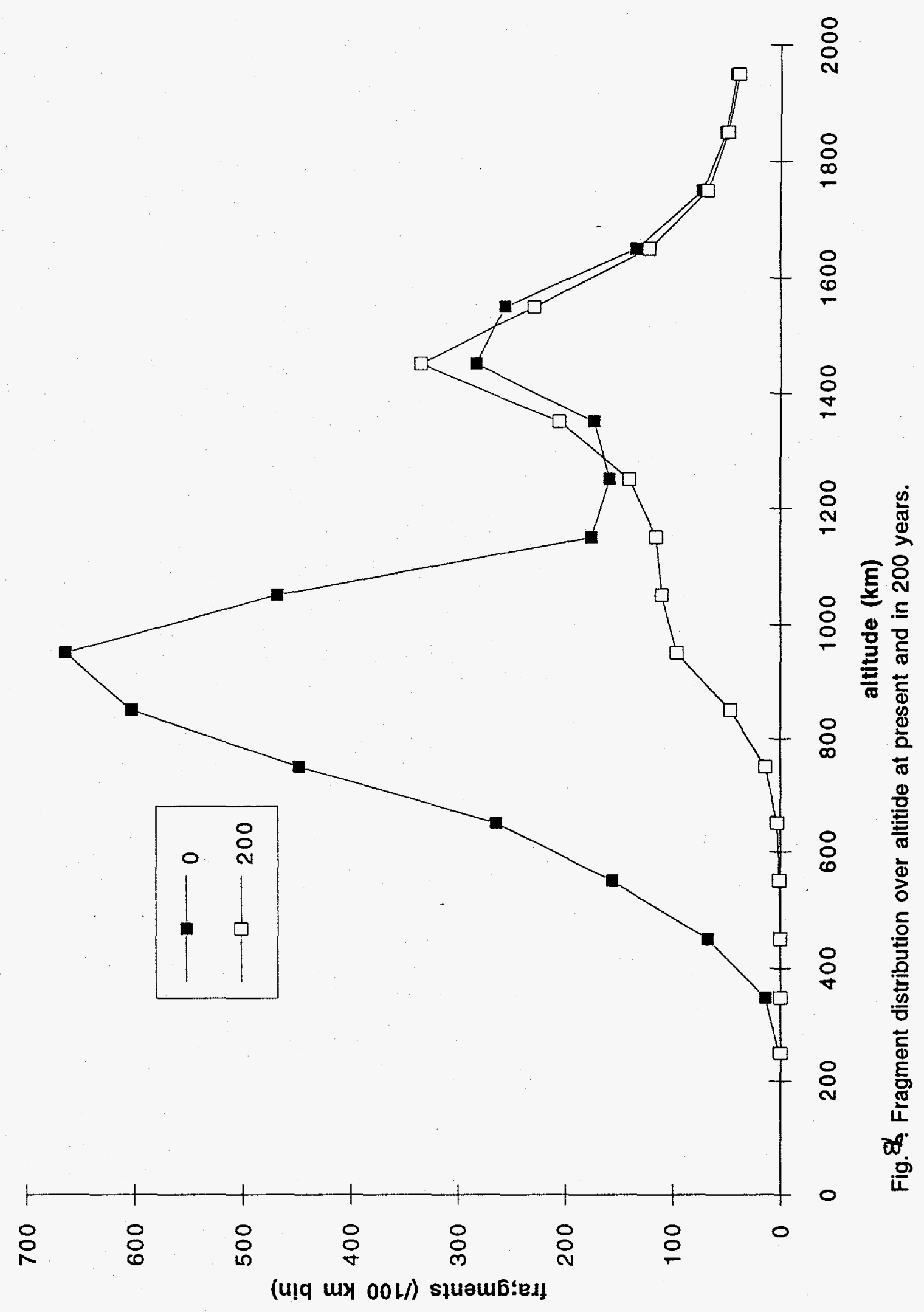


